\title{
BMJ open Maternal occupational exposure to asthmogens during pregnancy and risk of asthma in 7-year-old children: a cohort study
}

To cite: Christensen $\mathrm{BH}$, Thulstrup AM, Hougaard KS, et al. Maternal occupational exposure to asthmogens during pregnancy and risk of asthma in 7-year-old children: a cohort study. BMJ Open 2013;3:e002401. doi:10.1136/bmjopen-2012002401

- Prepublication history and additional material for this paper are available online. To view these files please visit the journal online (http://dx.doi.org/10.1136/ bmjopen-2012-002401).

Received 24 November 2012 Revised 27 February 2013 Accepted 4 March 2013

This final article is available for use under the terms of the Creative Commons Attribution Non-Commercial 2.0 Licence; see http://bmjopen.bmj.com

For numbered affiliations see end of article.

Correspondence to Dr Vivi Schlünssen; vs@mil.au.dk

\section{ABSTRACT}

Objectives: The objective of this study was to examine whether maternal exposure to asthmogens during pregnancy is associated with the development of asthma in 7-year-old Danish children, taking atopic status and sex into consideration.

Design: The study is a prospective follow-up of a birth cohort.

Setting and participants: A total of 41724 women and their children from The Danish National Birth Cohort were categorised according to maternal occupational exposure. Exposure information was obtained by combining job title in pregnancy and 18 months after pregnancy with a commonly used asthma Job Exposure Matrix.

\section{Primary and secondary outcome measures:}

Primary outcome was parent-reported asthma among their 7-year-old children in an internet-based questionnaire. Secondary outcome was asthma among the same children with or without atopic dermatitis and among boys and girls, respectively.

Results: Prenatal exposure to low molecular weight (LMW) agents was borderline associated with asthma in children with OR 1.17 (0.95 to 1.44$)$ for children with atopic dermatitis and $1.10(0.98$ to 1.22) for children without. Maternal postnatal exposure was associated with asthma (OR 1.15 (1.04 to 1.28). After mutual adjustment,postnatal exposure (OR 1.13 (0.99 to 1.29) and the combined effects of prenatal and postnatal exposure (OR 1.34 (1.19 to 1.51)) seem to increase the risk of asthma in children. No significant associations were observed for other prenatal or postnatal exposures. The gender of the child did not modify the aforementioned associations.

Conclusions: Maternal occupational exposures during pregnancy do not seem to be a substantial risk factor for the development of asthma in 7-year-old children. Maternal prenatal and postnatal exposures to LMW agents may predispose the propensity of the children to develop asthma. Future studies should prioritise the characterisation of the timing of exposure in relation to the birth.

\section{ARTICLE SUMMARY}

Article focus

- To study the impact of maternal occupationa exposure during pregnancy on the occurrence of asthma among their 7-year-old children.

Key messages

- A mother's occupational exposure during pregnancy is not a substantial risk factor for asthma in their 7-year-old children.

- Maternal prenatal and postnatal exposure to low-molecular-weight agents may increase the risk for asthma in 7-year-old children.

- Future studies should focus on the timing of exposure in relation to the birth.

Strengths and limitations of this study

- This study is the largest prospective study so far addressing the impact on childhood asthma of mothers' occupational exposure during pregnancy. It is also the first study where the atopic status of the child is taken into account when exploring the importance of prenatal occupational exposures on childhood allergic diseases.

- A substantial loss to follow-up was seen. We presume that the loss to follow-up did not influence our results to a major degree.

- The occupational exposure assessment was based on crude information on job titles and a general asthma Job Exposure Matrix.

- Definitions of asthma and atopy (defined as ever atopic dermatitis) were based on self-reported information from the mothers. Still, we were able confirm previously described associations between childhood asthma and several risk factors.

\section{BACKGROUND}

The occurrence of asthma, rhinitis and atopic dermatitis $(\mathrm{AD})$ has increased in the last decades, from a few per cent in the 1960s to $15-30 \%$ in the 1990 s. $^{1}{ }^{2}$ Lifestyle seems to influence the development of allergic diseases, also during childhood. ${ }^{3}$ Prenatal risk factors 
for asthma have been investigated and include environmental tobacco smoking, ${ }^{4}$ stress ${ }^{5}$ and maternal diet during pregnancy. ${ }^{6} 7$ Thus, the maternal environment during pregnancy might encompass risk factors for asthma in the child.

Allergic diseases are hallmarked by childhood immune dysfunction, but surprisingly, little attention has been paid to prenatal influences on immune development, although the fetal immune system is vulnerable to programming. ${ }^{8}$ In animals, airborne exposure to particles and aerosols of the mother during pregnancy have been shown to increase asthma susceptibility in the offspring, ${ }^{9} 10$ and findings in some human studies corroborate this association. ${ }^{11}{ }^{12}$ Maternal exposure to volatile organic compounds in pregnancy may skew the Th1/ Th2-immune response in the fetus, thereby potentially predisposing the children to asthma later in life. ${ }^{13}$

An increasing number of women work outside the home during pregnancy, and many are occupationally exposed to asthmogens, that is, chemical and biological agents that are recognised to increase the risk of workrelated asthma. It therefore seems pertinent to investigate whether women's working environments during pregnancy interfere with their children's propensity to develop asthma later in life. Two earlier cohort studies have investigated prenatal occupational exposures and the risk of asthma among adolescent ${ }^{14}$ and 0 -year-old to 7 -year-old children ${ }^{15}$ with inconclusive results.

Asthma can be characterised as allergic (atopic) or non-allergic (non-atopic). These two phenotypes are characterised by, for example, different risk factors. Endotoxin has, for example, opposite effects in atopic and non-atopic asthma. ${ }^{16}$ In the present study, we hypothesise that maternal exposure to asthmogens during pregnancy impacts on the fetal development of the immune system and predisposes the children to develop asthma later in life. Specifically, we analysed whether maternal occupational exposures during pregnancy are associated with the development of asthma in 7-year-old Danish children, taking atopic status into consideration.

\section{MATERIAL AND METHODS}

Population

The study population was part of the prospective cohort: The Danish National Birth Cohort (DNBC, 'Better health for mother and child'). The cohort was established in 1997-2002 to study pregnancy outcome and disease in children as a function of factors operating in early life. ${ }^{17}$ Women were recruited at their initial pregnancy consultation with their general practitioner or midwife and participation implied four telephone interviews; during weeks $12-16$ and weeks $30-35$ of pregnancy, and when the child was 6 and 18 months old. When the child was 7 years of age, the mothers answered an internet questionnaire on behalf of their children. Mothers without internet access were offered a paper version. The first telephone interview comprised questions about work, atopic diseases and risk factors for asthma (eg, smoking, use of medication and parity). The questionnaire at age 7 dealt with asthma, rhinitis and $\mathrm{AD}$ in the child and risk factors (eg, parental smoking)

A total of 100418 pregnancies were enrolled in the cohort. The first telephone interview was answered 92892 times. A total of 2396 women were no longer pregnant at the time of the interview and were excluded. Only singletons and only one pregnancy per woman (the first in the sampling period) were included, so 8720 siblings and 2963 children from multiple births were excluded. This left a total of 78813 mother/child pairs, of which the internet questionnaire at age 7 was answered for 45687 children. Among these, 3963 pairs were excluded due to lack of information on maternal occupational status. Thus, 41724 mother/child pairs were eligible for analysis.

\section{Occupational exposure}

Data on job exposure during pregnancy were obtained by combining reported information on job title (Danish International Standard classification of Occupations (DISCO)-codes) from telephone interview one during 12-16 weeks of pregnancy and a commonly used asthma Job Exposure Matrix (JEM) ${ }^{18}$ based on known risk factors for occupational asthma. The job titles were coded according to DISCO, the Danish edition of ISCO-88 (DISCO-code). Seven exposure categories were formed and consisted of the following occupations and typical allergens/irritants:

1. High-molecular-weight (HMW) agents (veterinarians, gardeners and bakers; grass pollen, animal dander, flour/grain);

2. Low-molecular-weight (LMW) agents or irritants (cooks, cleaners, hairdressers, dentistry and all kinds of manufacture of dust-producing materials; aerosols, dust from manufacture of wood/textile/stone/rubber/plastics etc);

3. Mixed HMW and LMW agents (healthcare professionals; antibiotics, latex, cleaning agents);

4. Farmers (organic dust, grass pollen, animal dander, ammonia);

5. Students (no or unknown exposure);

6. Unclassifiable (subjects where the same job title involved several different environments, eg, waiters, engineering technicians, biologists, shop managers; diverse exposure);

7. Reference (office workers, teachers and journalists; no or low exposure).

The applied JEM was modified slightly from $^{18}$ by Christensen $\mathrm{BH}$ and Schlünssen V to comply with the working conditions in Denmark based on an a priori knowledge of exposures to asthmogens in the Danish working environment. As an example, medical doctors were originally categorised as not being exposed to asthmogens, but were reclassified to the mixed group in the 
modified version of the JEM. Furthermore, farmers were identified as a special group as early life exposure to a farming environment has been shown to offer protection against allergic diseases. ${ }^{19}$ Also, students were considered as a special group due to the possible selection related to health.

Postnatal-occupational exposure groups were established using the four-digit DISCO-codes 18 months after the birth of the child, that is, at a time when maternity leave was terminated and the mother would most probably have returned to work. The codes were retrieved from Statistics Denmark, as every adult person ( $\geq 18$ years) in Denmark is classified annually according to occupation. Maternal postnatal occupation was categorised in the same JEM categories as for the prenatal exposures. Women classified as students during pregnancy, but without a DISCO-code indicative of employment after having given birth, were not included in the postnatal analyses.

The level of education and socioeconomic status (SES; self-employed, employees at the highest level, employees, students, unemployed or not in the workforce) were obtained for both the father and the mother (the calendar year preceding the birth year of the child) from Statistics Denmark. Information on the parent with the highest level of education/SES defined the level for the family.

\section{Outcome}

We used validated core questions on asthma from the International Study of Asthma and Allergies in Childhood. ${ }^{20}$ Asthma in the child at age 7 was defined as an affirmative answer to one or more of three questions: 'Has your child had wheezing or whistling in the chest in the last 12 months?'; 'Has your child ever had asthma?' or to 'Has your child ever been diagnosed with asthma by a doctor?'

Ever AD was used as a proxy for atopy among the children. Information on $\mathrm{AD}$ was collected by a telephone interview when the child was 18 months old and by questionnaire at age 7 . It was defined as a parental report at 18 months of $\mathrm{AD}$ ever AND itchy rash in the locations known to be typical for AD AND/OR a report of persistent itchy rash in the locations known to be typical for $\mathrm{AD}$ at age 7 .

\section{Confounders}

Information on the known possible confounders according to prior knowledge from other studies on the effects of asthma in children was obtained primarily from the telephone interview at 12-16 weeks of gestation: maternal age, prepregnancy body mass index (BMI), parity, smoking during pregnancy and furry animals in the home. Maternal atopic disposition was defined as ever reported asthma, rhinitis and/or AD. Information on the maternal use of acetyl salicylic acid, paracetamol, folic acid and antibiotics during pregnancy was retrieved from the second telephone interview at 30-35 weeks of gestation. Birth weight, gestational age at birth, sex and singleton status were obtained from the Danish Birth Registry.

\section{Statistical methods}

All analyses were performed in STATA SE V.12.0 (STATA Corp., Texas, USA). Univariate analyses were performed for categorical variables using $\chi^{2}$ tests. For comparisons of continuous variables, the independent sample t test or the Kruskal-Wallis test was used. A multivariate unconditional logistic regression analysis was used to evaluate associations between exposure and outcome.

A univariate analysis revealed an equal distribution between the exposure groups for some of the potential confounders, which was subsequently excluded from the model. The final model contained the following maternal variables: age (16-24; 25-29; 30-35; 35+ years), prepregnancy BMI ( $\leq 19 ; 20-24 ; 25-29 ; 30+)$, atopy (yes/no), smoking during pregnancy (no; $1-10 ; 10+$ cigarettes/day), use of medication during pregnancy (yes/no to the use of acetyl salicylic acid, paracetamol, folic acid and antibiotics), parity $(0 ; 1-2 ; 3+)$ and furry animal ownership during pregnancy (yes/no). For the children, small for gestational age (SGA) (yes/no) and sex were included. SGA was defined as the smallest $10 \%$ of the children born in a specific gestational week based on birth weights from the entire BSMB cohort, and was calculated separately for boys and girls. The analysis was repeated after including the children's exposure to environmental tobacco smoke (ETS) in the model according to the information on the parents' current smoking. Unless otherwise stated, the level of significance was $\mathrm{p}<0.05$, two-sided.

\section{RESULTS}

Table 1 shows the demographic characteristics of the cohort stratified by exposure groups. Women had a mean age of 30 years at inclusion. Students were younger than the other groups, that is, $74.3 \%$ were below 30 years of age. About half $(52.1 \%)$ of the women had not given birth previously. Among the students, $66.6 \%$ were primiparous. On average, $45.8 \%$ of the women had used paracetamol, most prevalent in the mixed exposure group (52.3\%) and least prevalent in the farmer group (39.6\%). A majority $(79.4 \%)$ of the women were non-smokers during pregnancy. The LMW group had the highest tobacco use with $17.4 \%$ smoking $10+$ cigarettes/day. A similar pattern was seen at follow-up at 7 years of age, but in general, fewer mothers smoked at follow-up, with a decrease from one in four to one in six. The lowest prevalence of atopy $(15.2 \%)$ was seen in the HMW group, whereas the prevalence was highest among students (23.8\%).

Online supplementary table E1 presents the characteristics among participants and non-participants. The distributions of exposure groups were quite similar between participants and non-participants, but with a slightly lower number of LMW exposed and students among the 
Table 1 Demographics, categorised according to mothers' exposure during pregnancy into seven categories: high-molecular weight (HMW), low-molecular weight/irritants (LMW), mixed (a mixture of HMW and LMW), farmers, unclassifiable, students and references

\begin{tabular}{|c|c|c|c|c|c|c|c|c|}
\hline $\begin{array}{l}\text { During pregnancy } \\
\text { Mother }\end{array}$ & $\begin{array}{l}\text { HMW } \\
\mathrm{n}=409\end{array}$ & $\begin{array}{l}\text { LMW } \\
n=3705\end{array}$ & $\begin{array}{l}\text { Mixed } \\
\mathrm{n}=7722\end{array}$ & $\begin{array}{l}\text { Farmer } \\
n=330\end{array}$ & $\begin{array}{l}\text { Unclassifiable } \\
n=2405\end{array}$ & $\begin{array}{l}\text { Student } \\
\mathrm{n}=3867\end{array}$ & $\begin{array}{l}\text { Reference } \\
\mathrm{n}=23286\end{array}$ & $\begin{array}{l}\text { Missing } \\
(\%)\end{array}$ \\
\hline Age, years, median (10\%; 90\%) & $29(24 ; 35)$ & $29(24 ; 35)$ & $30(25 ; 36)$ & $30(24 ; 36)$ & $31(26 ; 36)$ & $27(23 ; 33)$ & $30(26 ; 36)$ & 0 \\
\hline BMI, median (10\%; $90 \%)$ & $23.0(19.5 ; 31.6)$ & $23.1(19.4 ; 30.5)$ & $22.6(19.4 ; 29.0)$ & $22.7(19.7 ; 28.4)$ & $22.3(19.4 ; 27.3)$ & $22.0(19.1 ; 27.5)$ & $22.4(19.4 ; 28.2)$ & 1.6 \\
\hline Parity & & & & & & & & 0.1 \\
\hline 0 & 50.6 & 48.2 & 48.6 & 45.3 & 53.9 & 66.6 & 51.5 & \\
\hline 1 or 2 & 46.5 & 49.5 & 48.0 & 48.0 & 44.6 & 31.8 & 46.7 & \\
\hline $3+$ & 2.9 & 2.4 & 3.4 & 6.7 & 1.5 & 1.6 & 1.8 & \\
\hline \multicolumn{9}{|l|}{ Use of medicine } \\
\hline Acetyl Salicylic Acid & 4.9 & 4.5 & 4.1 & 4.9 & 5.2 & 4.4 & 4.3 & 5.4 \\
\hline Paracetamol & 45.4 & 47.2 & 52.3 & 39.6 & 41.3 & 43.4 & 44.3 & 5.4 \\
\hline Antibiotics & 19.1 & 16.5 & 18.8 & 14.9 & 17.5 & 17.7 & 17.3 & 5.3 \\
\hline Folic acid & 55.5 & 56.0 & 56.1 & 52.1 & 55.4 & 58.3 & 58.3 & 5.2 \\
\hline Rural residence & 15.4 & 8.0 & 7.4 & 44.2 & 6.4 & 3.9 & 5.6 & 0.0 \\
\hline Furry animals* & 63.6 & 49.1 & 41.4 & 67.6 & 34.6 & 29.6 & 35.5 & 0.0 \\
\hline Smoking & & & & & & & & 0.0 \\
\hline No use of tobacco & 75.3 & 67.1 & 77.3 & 82.1 & 85.3 & 79.1 & 81.5 & 0.0 \\
\hline $1-10$ cigarettes/day & 9.5 & 15.4 & 11.8 & 7.9 & 8.0 & 10.9 & 10.4 & 0.0 \\
\hline$>10$ cigarettes/day & 15.2 & 17.4 & 10.9 & 10.0 & 6.7 & 10.0 & 8.2 & 0.0 \\
\hline Atopy $^{\dagger}$ & 15.2 & 19.9 & 22.1 & 17.3 & 21.7 & 23.8 & 21.0 & 0.0 \\
\hline \multicolumn{9}{|l|}{ Child } \\
\hline Boy & 50.1 & 51.1 & 51.5 & 50.3 & 51.7 & 51.8 & 51.2 & 0.0 \\
\hline Birth weight, kg, median & $3.60(2.90 ; 4.30)$ & $3.58(2.88 ; 4.25)$ & $3.60(2.95 ; 4.25)$ & $3.65(2.95 ; 4.29)$ & $3.60(2.98 ; 4.26)$ & $3.58(2.95 ; 4.21)$ & $3.60(2.95 ; 4.25)$ & \\
\hline \multicolumn{9}{|l|}{$(10 \% ; 90 \%)$} \\
\hline Small for gestational age & 9.6 & 10.8 & 8.7 & 7.9 & 8.5 & 9.7 & 8.7 & 0.3 \\
\hline \multicolumn{9}{|l|}{ At 7-year follow-up } \\
\hline Maternal smoking & 19.3 & 27.5 & 17.5 & 13.9 & 10.7 & 16.1 & 15.1 & 0.0 \\
\hline Paternal smoking & 27.9 & 31.1 & 22.8 & 18.5 & 18.2 & 21.5 & 20.4 & 0.0 \\
\hline $\begin{array}{l}\text { Child atopy defined as ever } \\
\text { atopic dermatitis }\end{array}$ & 16.6 & 16.5 & 19.0 & 17.3 & 18.6 & 18.1 & 18.1 & 0.0 \\
\hline Child ETS exposure & 37.4 & 43.6 & 31.5 & 26.4 & 23.8 & 30.0 & 28.1 & 0.0 \\
\hline
\end{tabular}

${ }^{*}$ Ownership of furry animals during pregnancy.

†Atopic status of the women.

The numbers are presented as \% unless otherwise stated.

BMI, body mass index; ETS, environmental tobacco smoke. 
Table 2 Unadjusted prevalence of asthma stratified for atopy defined as ever atopic dermatitis (AD) in the 7-year-old children $(n=41724)$

\begin{tabular}{|c|c|c|c|}
\hline Exposure group & All children n (\%) & AD children $n(\%) \dagger$ & Non-AD children $n(\%) \dagger$ \\
\hline High molecular & $72(17.7)$ & $18(26.5)$ & $54(15.8)$ \\
\hline Low molecular/irritants & $689(18.6)^{*}$ & $173(28.3)^{\star *}$ & $516(16.7)^{\star \star \star}$ \\
\hline Mixed & $1264(16.4)$ & $348(23.8)$ & $916(14.6)$ \\
\hline Farmer & $43(13.0)$ & $14(24.6)$ & 29 (10.6) \\
\hline Unclassifiable & $360(15.0)$ & 98 (21.9) & $262(13.4)$ \\
\hline Student & $627(16.2)$ & $161(23.0)$ & $466(14.7)$ \\
\hline Reference & 3553 (15.3) & 934 (22.2) & 2619 (13.7) \\
\hline Total n (\%) & $6608(15.8)$ & $1746(23.1)^{\star \star \star \star}$ & $4862(14.2)$ \\
\hline
\end{tabular}

participating mothers compared with the nonparticipating mothers. More of the participating mothers were non-smokers during pregnancy, $79 \%$ versus $73 \%$; they were slightly older (30 vs 29 years), better educated (academic level, $34 \%$ vs $25 \%$ ), belonged to a higher socioeconomic group (employees at the highest level, $25 \%$ vs $18 \%$ ), and more were expecting their first child compared with the non-participants, $52 \%$ vs $49 \%$. Maternal atopic status was similar in the two groups. At birth, children of participating mothers, on average, weighed $30 \mathrm{~g}$ more and the number of SGA children was $9 \%$ in participants compared with $10.7 \%$ among non-participants.

Table 2 shows the prevalence of asthma stratified for atopic status defined as ever $\mathrm{AD}$. The overall prevalence of asthma was 16\%; $23 \%$ for $\mathrm{AD}$ and $14 \%$ for non-AD children. Children from the LMW and the HMW-exposure groups had the highest prevalence of asthma, both in total $(18-19 \%)$, and for the AD group $(27-28 \%)$ and non-AD group (16-17\%). Farmers' children had the lowest prevalence of asthma, which was not significantly different from that of the other exposure groups. In online supplementary table E2, the prevalence of asthma was further stratified by sex. The prevalence of asthma was higher among boys (19\%) compared with girls $(13 \%)$, and for both sexes the overall prevalence of asthma was higher for AD compared with non-AD children. Children from the LMW and HMW exposure groups had the highest prevalence of asthma in boys; for girls, this was only true in respect of the LMW exposure group.

Table 3 shows the crude and adjusted ORs for the associations between mothers' occupational exposure during pregnancy and asthma in the child. In model 2, levels of education and SES were added to the a priori chosen confounders. For HMW agents, ORs were nonsignificantly increased in the range 1.26-1.44 in both the crude and the adjusted analyses, especially for AD children. Children of mothers exposed to LMW agents during pregnancy had an increased OR for asthma in the crude analysis for both AD (OR 1.38 (95\% CI 1.14 to 1.67$)$ ) and non-AD children (OR 1.26 (1.13 to 1.39 ). The same tendency was seen in model 1 with OR 1.17 (0.95 to 1.44$)$ for $\mathrm{AD}$ and 1.10 (0.98 to 1.22$)$ for non-AD children. The ORs were further decreased in model 2 with $\mathrm{OR} 1.10$ (0.88 to 1.37$)$ for $\mathrm{AD}$ and 1.03 (0.91 to 1.15) for non-AD children.

For the other exposure groups, no significant association between maternal exposure and asthma was observed in the children. In the crude analysis, a borderline significant association between being in student/ mixed groups and asthma was seen with OR 1.05-1.08, but without confirmation in model 1 or 2 .

The analysis in table 3 was repeated taking the 7-year-old children's current parental ETS into account. This did not change the results (data not shown). The same was true when paternal atopic status was included.

Even though educational level and SES were considered as potential confounders, they were correlated to our exposure variable, partly because the DISCO-code was included in the definition of SES. We therefore decided to use model 1 in the ensuing analysis. We did not find any significant interactions between exposure and ever $\mathrm{AD}$ when the interaction exposure*ever $\mathrm{AD}$ was included in the model.

Model 1 was repeated after stratification for sex; please see online supplementary table E3. In general, similar results were found for boys and girls, that is, children of mothers exposed to LMW agents during pregnancy had a borderline increased OR for asthma in both girls (1.14 (0.98 to 1.32$))$ and boys (1.08 (0.95 to 1.23$))$. For girls, the association was significant for $\mathrm{AD}$ children, 1.43 (1.09 to 1.88 ), whereas for boys this was true for non-AD children, 1.23 (1.04 to 1.46 ).

In online supplementary table E4, ORs for covariates in model 1 are displayed with asthma as the outcome of interest. The mother's age was inversely associated to asthma in the child. The children of women $<25$ years of age had an increased OR for asthma, 1.40 (1.27 to 1.55), compared with 25-year-old to 29-year-old women (reference). The risk was further decreased for 30-34 years, OR 0.85 (0.79 


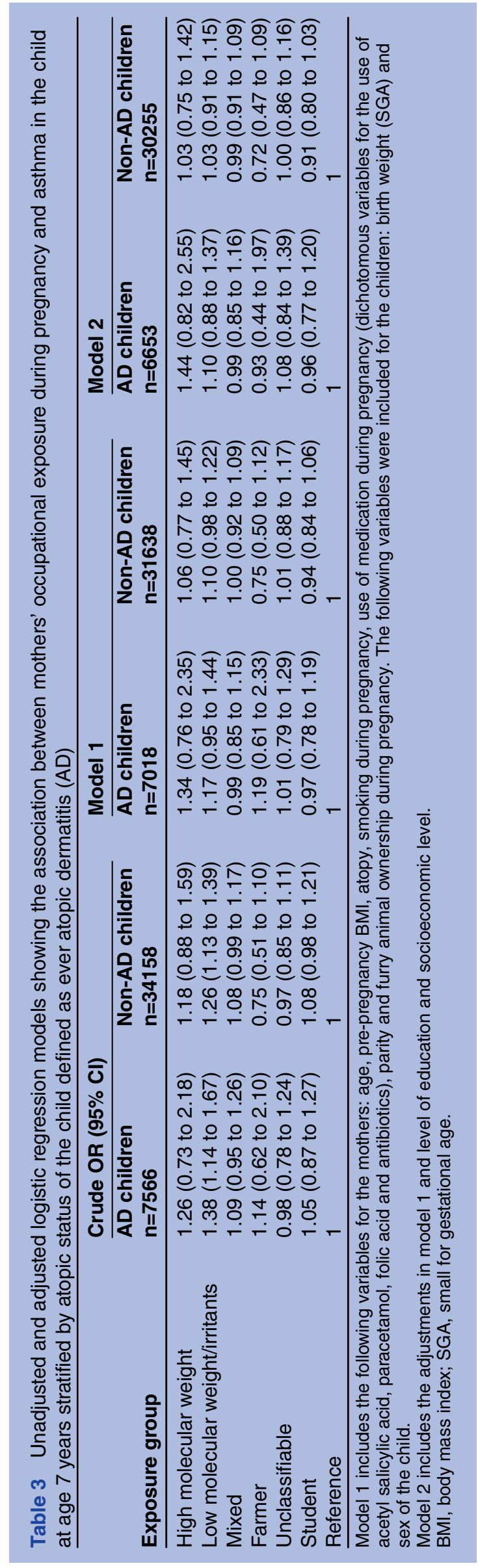

to 0.91 ) and $35+$ year-old women, OR 0.77 (0.70 to 0.84 ). Maternal pre-pregnancy BMI was positively associated with asthma with ORs, 1.17 (1.09 to 1.26) and 1.35 (1.21 to 1.49) for BMI 25-29 and above 30, respectively, compared with BMI 20-24 (reference). Children with maternal atopic disposition had increased OR for asthma (1.69 (1.59 to 1.80$)$ ). The use of paracetamol and antibiotics during pregnancy was associated with asthma in the children, OR 1.08 (1.02 to 1.14 ) and 1.17 (1.09 to 1.26), respectively. Smoking during pregnancy was positively associated with asthma in a dose-dependent manner with OR 1.20 (1.10 to 1.31) for 1-10 cigarettes/day), and OR 1.42 (1.30 to 1.55 ) for $>10$ cigarettes/day compared with nonsmokers (reference). Parity was positively associated with asthma, with OR 1.14 (1.07 to 1.21) for 1-2 pregnancies and OR 1.19 (0.97 to 1.46) for three or more pregnancies compared with no earlier pregnancy (reference), whereas pet ownership was not associated with childhood asthma, OR 0.97 (0.92 to 1.03).

Being a boy and being born SGA were both positively associated with asthma with ORs 1.57 (1.48 to 1.66) and 1.17 (1.07 to 1.29$)$, respectively.

The analysis in online supplementary table E2 was repeated after stratification for atopic status in the children, and almost identical results were seen for $\mathrm{AD}$ and non-AD children (data not shown).

Figure 1 shows the adjusted logistic regression analysis on the association between mothers' prenatal and postnatal exposure and asthma in the children. For LMW agents, both prenatal and postnatal exposure were significantly associated with asthma in the children, ORs

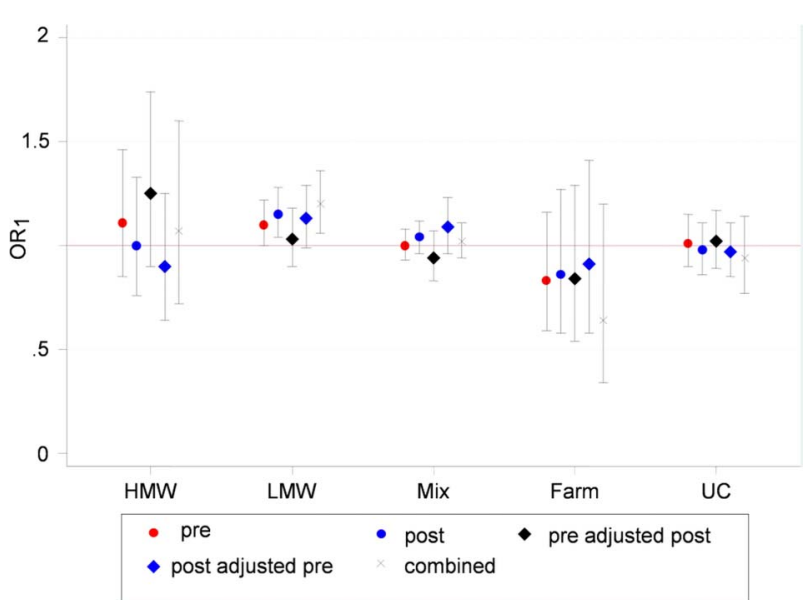

Figure 1 Adjusted logistic regression analysis on the association between mothers' prenatal and postnatal exposure and asthma in 7-year-old children. Reference: reference workers. Pre, prenatal exposure; post, postnatal exposure; post just pre, postnatal exposure adjusted for prenatal exposure; pre just post, prenatal exposure adjusted for postnatal exposure; combined, additive effect of prenatal and postnatal exposure. Included in the model: maternal age, pre-pregnancy $\mathrm{BMI}$, atopy, smoking during pregnancy, use of medication during pregnancy, furry animal ownership during pregnancy, SGA and sex of the child. 
1.10 (1.00 to 1.22 ) and 1.15 (1.05 to 1.28 ), respectively. After mutual adjustments, only postnatal exposure (OR 1.13 (0.99 to 1.29)) and the combined effects of prenatal and postnatal exposure (OR 1.34 (1.19 to 1.51)) were associated with asthma in the child. For the other exposure groups, no significant associations were seen for either prenatal or postnatal exposure.

The association between maternal-specific occupations (included in the LMW exposure group) and asthma in the children is displayed in table 4 . In the adjusted analysis, hairdressing was borderline associated with asthma in the child, OR 1.29 (0.99 to 1.69 ), without any significant difference between $\mathrm{AD}$ and non-AD children. For $\mathrm{AD}$ children, cleaning was borderline associated with asthma with OR 1.56 (0.98 to 2.47). A similar pattern was observed for non-AD children and mother's work in the chemical industry, OR 1.54 (0.97 to 2.45).

The above analysis was repeated after stratification for sex; please see online supplementary table E5. AD boys of mothers working as cleaners during pregnancy (2.29 (1.24 to 4.26) and non-AD girls of women working in the chemical industry (OR 2.15 (1.12 to 4.09) had increased OR for asthma.

\section{DISCUSSION}

\section{Key findings}

We found that maternal occupational exposure to LMW agents during and after pregnancy may be associated with asthma in 7-year-old children. Subanalyses suggested associations between a few specific LMW jobs (hairdressing, cleaning and chemical industry) and asthma in the children. Maternal prenatal and postnatal LMW exposures were associated with childhood asthma, but after mutual adjustments, postnatal exposure seemed more significant.

\section{How do the findings fit with earlier study results?}

In a Swedish multigenerational register study, 47000 1-year-old to 10-year-old children with a first hospitalisation for asthma were identified. ${ }^{21}$ The children were linked to national census data on parental (maternal if available, otherwise paternal) occupation prior to the birth of the child. Standardised incidence ratios (SIRs) of 1.20 or higher were found for hospitalisation due to asthma for boys of beverage manufacturers, packers/ loaders, cooks/stewards, home helpers and building caretakers/cleaners. We confirmed that atopic boys of cleaners had an increased OR for asthma. For girls, SIRs of 1.20 or higher were found for chemical process workers, cooks/stewards and waiters. In the present study, we could confirm adjusted increased ORs for asthma in 7-year-old girls of chemical workers.

Two previous birth cohort studies investigated prenatal occupational exposures and the risk of asthma and wheeze in children. In a study among 7844 14-year-old to 28-year-old adolescents, Magnusson $e$ a $\mathrm{ll}^{14}$ concluded that some maternal jobs (eg, bakers, dental assistants, technicians, librarians and bookbinders) during pregnancy were associated with an elevated risk of wheeze or asthma in the children.

\section{Prenatal versus postnatal effects}

In a cohort of 14000 children from the ALSPAC study, Tagiyeva $e t a l^{15}$ found that maternal occupational exposure to latex and biocides/fungicides was associated with an increased likelihood of asthma in 7-year-old children. Adjusting for maternal postnatal exposure made the prenatal effects disappear. Furthermore, paternal occupational exposure to flour dust after birth was associated with an increased likelihood of childhood wheeze and asthma.

These findings are in line with our results, indicating that postnatal maternal exposure seems to influence childhood asthma more than prenatal exposure. These results should, however, be interpreted with caution since prenatal and postnatal exposures are closely correlated. Most mothers worked within the same occupation during the toddler period, that is, only one-fifth of the included women changed the occupational exposure group from early pregnancy and until 18 months after childbirth. The effect of postnatal parental occupation on childhood asthma has been suggested to occur due to a 'carrier home' effect, ${ }^{22}$ implicating that the parents carry home components of the work environment leading to the indirect exposure of the child from the parental work environment. Effects of maternal exposure during pregnancy are also biologically plausible. Antigens may be transferred from the placenta or via the amniotic fluid and cause the sensitisation of the fetus to take place. ${ }^{1123}$ Allergen exposure during pregnancy can also promote an excessive or prolonged Th2-skewed immunity after birth. This exaggerates the normal dominance of Th2 during and after pregnancy, and increases the propensity of developing allergy. ${ }^{9} 24$

Also, other postnatal modifying factors are to be considered, such as breastfeeding, early-life day care, number of siblings and ETS. In our study, only $0.2 \%$ of the women had never breastfed their children, and more than $90 \%$ of the children attended day care from a very early age, making it unlikely that these potential risk factors have influenced our results considerably. Adjusting for postnatal ETS and parity did not change our estimates.

\section{Implications of atopic status and sex of the child}

As far as we know, this is the first study to take atopic status in the children into consideration when studying the impact of maternal work on asthma in children. We did not find convincing differences between $\mathrm{AD}$ and non-AD children for associations between maternal occupational exposures and asthma in children. The present study thus corroborates the results from our study on hay fever ${ }^{25}$ in the same mother/child pairs. We did, however, notice non-significantly increased ORs for $\mathrm{AD}$ children in mothers exposed to HMW agents and 


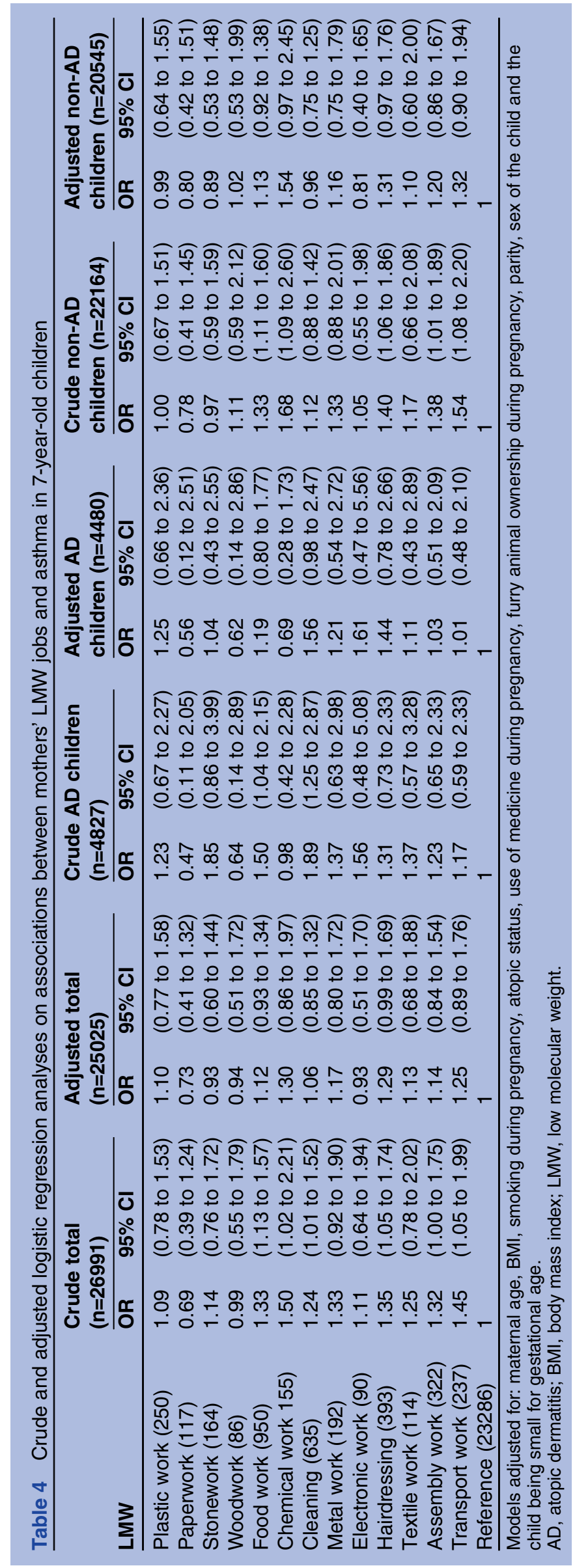

mothers with cleaning jobs compared with ORs close to 1 for the non-AD children. We also noticed an insignificantly increased OR for non-AD children among mothers working in the chemical industry compared with $\mathrm{AD}$ children.

The impact of atopic status on the associations between environmental exposures and asthma has been emphasised before, that is, others have found that exposure to endotoxin early in life may protect against the development of atopic asthma, but not non-atopic asthma. ${ }^{16} 26$ Exposure to maternal domestic cleaning has been found to increase the risk for persistent wheeze only in nonatopic children in the ALSPAC study. ${ }^{12}$

As expected, we found a higher prevalence of asthma among boys compared with girls, but in general, sex did not modify the effect of maternal work during pregnancy on the development of offspring asthma. In a few analyses stratified by both $\mathrm{AD}$ and sex, differences were found for boys and girls, but our results are too vague to support a specific gender effect.

\section{Implications of SES}

Even though educational level and SES were considered as potential confounders, they were correlated to our exposure variable, and furthermore, SES and educational level were positively associated with asthma with OR 2.11 (1.53-2.92) for asthma among the unemployed compared with the self-employed and OR $0.56(0.52$ 0.61 ) for women with an academic education compared with compulsory schooling. We therefore decided to exclude educational level and SES from the final model due to risk for overadjustment.

\section{Strengths and limitations of the study}

To our knowledge, the present study is the largest of its kind so far, regarding maternal occupational exposure to asthmogens during pregnancy and asthma in the children. The DNBC data collection was performed prospectively. Our results confirm previously described associations between childhood asthma and several risk factors, that is, maternal atopic disposition, ${ }^{27}$ prepregnancy BMI ${ }^{28}$ age, ${ }^{29}$ gestational smoking, ${ }^{30}$ use of medication, ${ }^{31} 32$ as well as offspring sex ${ }^{33}$ and SGA. ${ }^{34}$

A number of limitations have to be considered as well. In the DNBC cohort, non-smoking, older and well-educated women are over-represented compared with the general Danish population. ${ }^{35}$ A substantial loss to follow-up is present, that is, nearly $60 \%$ of the original cohort was not included in our analyses, and only 53\% (41 724 of 78813 eligible mother/child pairs) were included in the current analysis. We found the same characteristics among the loss to follow-up subjects in the current study compared with the DNBC cohort in general, that is, more of the participants were better educated, smoked less and belonged to a higher socioeconomic group. Otherwise, only small differences were seen according to age, parity and birth weight. For more outcomes, for example, childhood asthma, register data have indicated that associations in DNBC are not 
biased by non-participation. ${ }^{35}{ }^{36}$ Furthermore, we have adjusted for some of the obvious confounders in the analysis, and we found similar associations between maternal exposure and asthma for smokers and non-smokers.

The asthma prevalence found in this study is comparable to other Danish prevalence data on childhood asthma with a similar asthma definition. ${ }^{37}$ We are aware that mothers with allergic diseases may avoid certain types of occupations, and employees with work-related asthma or allergy may be advised to avoid or reduce occupational exposure leaving non-atopics in risk occupations (healthy worker effect). However, the proportion of women with atopy did not differ between the exposure groups (except in students) and did not differ between participants and non-participants. All in all, we presume that the loss to follow-up did not influence our results to a major degree, but it cannot be excluded that it has attenuated or overestimated the associations presented to some extent.

The occupational exposure assessment was based on crude information on job titles and the overall probability of exposures for each job type was based in a specific asthma JEM. This lack of access to detailed individual exposure information implies a substantial risk of non-differential exposure misclassification. Furthermore, we have job information from only one time point in pregnancy (12-16 week), and a change in job during pregnancy was not accounted for, which might add to the exposure misclassification as well. Based on information about postnatal exposure, where only one-fifth of the women changed jobs between 12 and 16 weeks of pregnancy and 18 months after birth, we expect that relatively few women change jobs during pregnancy, but we cannot say that with certainty.

Prenatal exposures were derived from the interviewbased job descriptions, where postnatal codes originated from register data from Statistics Denmark based on obligatory information handed in by the employer to the Danish tax authorities. Some inconsistency between the two data sources are to be expected. The selfreported data are probably more precise, but also prone to a possible reporting bias. As the exposure group was identical in the interview and the register data for more than $80 \%$, we do not think the different data sources had a major impact on the result.

Another clear limitation of this epidemiological study is the lack of specific measures for atopy, for example, positive skin prick tests or specific IgE. Ever AD in the children was used as a proxy for atopy, as this condition is relatively closely linked to elevated serum $\operatorname{IgE}$ and often also to specific sensitisation. ${ }^{38}$ Misclassification is, however, inherently present. Many atopic subjects (children and adults), defined by a positive Skin Prick Test or elevated serum IgE, do not have $\mathrm{AD}$ but have other manifestations of allergy, and atopy does not always result in $\mathrm{AD}$. It can be argued that allergic rhinitis is even more closely related to IgE-mediated sensitisation than $\mathrm{AD}$, but owing to the age of the children ( 7 year), only a few have developed allergic rhinitis, and therefore allergic rhinitis was not a good predictor for atopy in this study. Still, with these weaknesses in mind, we think it is justified to use the presence of $\mathrm{AD}$ as a reasonable proxy for atopy among young children.

We defined $\mathrm{AD}$ by a combination of maternally reported $\mathrm{AD}$ and eczema in locations typical for $\mathrm{AD}$. These questions have been validated against a clinical investigation for $\mathrm{AD}$ and were deemed suitable for defining $\mathrm{AD}$ in large-scale epidemiological studies. ${ }^{39}$ Our definition is not entirely identical to the wording suggested by Benn $e t a l^{89}$, eczema at 7 years was also included and a slightly different combination of questions applied. Overall, this was expected to increase the sensitivity at the expense of decreased specificity.

Diagnosis of asthma in the children was based on maternal reports. Although standardised and validated asthma questions were used, ${ }^{20}$ some non-differential misclassification would still be expected, and our results might underestimate a true effect.

\section{CONCLUDING REMARKS}

Our study aimed to add to the limited knowledge currently available on the impact of prenatal maternal occupational exposure and asthma in children. It was based on a large population cohort of women and their children. The women had a variety of occupational job titles, making exposure contrast possible, even with a trivial exposure misclassification.

In conclusion, maternal occupational exposures during pregnancy do not seem to be a substantial risk factor for the development of asthma in 7-year-old children. Maternal occupational exposure to LMW agents early in the child's life may predispose them to asthma. Future studies should prioritise exposure characterisation during both the prenatal and postnatal periods in order to disentangle the importance of the timing of maternal exposure for development of asthma in children.

\section{Author affiliations}

${ }^{1}$ Department of Public Health, Section of Environment, Occupation and Health, Danish Ramazzini Centre, Aarhus University, Aarhus, Denmark ${ }^{2}$ Department of Occupational Medicine, Danish Ramazzini Centre, Aarhus University Hospital, Aarhus, Denmark

${ }^{3}$ The National Research Center for the Working Environment, Copenhagen, Denmark

${ }^{4}$ Department of Occupational Medicine, Hospital of South-West Jutland, Esbjerg, Denmark

${ }^{5}$ Department of Paediatrics, University Hospital Herlev, Herlev, Denmark

${ }^{6}$ Department of Public Health, Section of Biostatistics, Aarhus University, Aarhus, Denmark

Contributors All authors planned the study and performed the analysis plan together. BHC and VS were the lead writers. MF and BHC were the primary analysts. All authors interpreted the data and critically revised successive drafts of the manuscript. All authors read and approved the final manuscript.

Funding This study is part of the Danish collaborative MINERVA project (www. minervanet.dk) addressing occupational risks to human reproduction, supported by a grant from the Danish Working Environment Research Fund (contract 20080016458). Additional funding was obtained from Aarhus University and by the Danish Graduate School of Public Health Science (GRASPH).

Competing interests None. 
Ethics approval The study was a register study based on data collected earlier. The original DNBC study has an approval from the Ethics Committee. This study was approved by the Danish data protection agency.

Provenance and peer review Not commissioned; externally peer reviewed.

Data sharing statement Documentation for how the analysis has been performed can be provided by contacting the corresponding author. The data have been analysed by the authors on a data platform in Statistics Denmark where the register data and DNBC data were merged after an application on the specific register variables of interest was approved by Statistics Denmark.

\section{REFERENCES}

1. James AL, Knuiman MW, Divitini ML, et al. Changes in the prevalence of asthma in adults since 1966: the Busselton health study. Eur Respir J 2010;35:273-8.

2. Eder W, Ege MJ, von Mutius E. The asthma epidemic. N Engl J Med 2006;355:2226-35.

3. Asher MI, Montefort S, Bjorksten B, et al. Worldwide time trends in the prevalence of symptoms of asthma, allergic rhinoconjunctivitis, and eczema in childhood: ISAAC phases one and three repeat multicountry cross-sectional surveys. Lancet 2006;368:733-43.

4. Alati R, AI MA, O'Callaghan M, et al. In utero and postnatal maternal smoking and asthma in adolescence. Epidemiology 2006;17:138-44.

5. Wright RJ. Prenatal maternal stress and early caregiving experiences: implications for childhood asthma risk. Paediatr Perinat Epidemiol 2007;21(Suppl 3):8-14.

6. Haberg SE, London SJ, Stigum H, et al. Folic acid supplements in pregnancy and early childhood respiratory health. Arch Dis Child 2009;94:180-4

7. Willers SM, Wijga $A H$, Brunekreef $B$, et al. Maternal food consumption during pregnancy and the longitudinal development of childhood asthma. Am J Respir Crit Care Med 2008;178:124-31.

8. Dietert RR, Zelikoff JT. Early-life environment, developmental immunotoxicology, and the risk of pediatric allergic disease including asthma. Birth Defects Res B Dev Reprod Toxicol 2008;83:547-60.

9. Fedulov AV, Leme A, Yang Z, et al. Pulmonary exposure to particles during pregnancy causes increased neonatal asthma susceptibility. Am J Respir Cell Mol Biol 2008;38:57-67.

10. Hamada K, Suzaki $Y$, Leme A, et al. Exposure of pregnant mice to an air pollutant aerosol increases asthma susceptibility in offspring. $J$ Toxicol Environ Health A 2007;70:688-95.

11. Peters JL, Suglia SF, Platts-Mills TA, et al. Relationships among prenatal aeroallergen exposure and maternal and cord blood IgE: project ACCESS. J Allergy Clin Immunol 2009;123:1041-6.

12. Henderson J, Sherriff A, Farrow A, et al. Household chemicals persistent wheezing and lung function: effect modification by atopy? Eur Respir J 2008;31:547-54.

13. Lehmann I, Thoelke A, Rehwagen M, et al. The influence of maternal exposure to volatile organic compounds on the cytokine secretion profile of neonatal T cells. Environ Toxicol 2002;17:203-10.

14. Magnusson LL, Wennborg $\mathrm{H}$, Bonde JP, et al. Wheezing, asthma, hay fever, and atopic eczema in relation to maternal occupations in pregnancy. Occup Environ Med 2006;63:640-6.

15. Tagiyeva N, Devereux G, Semple S, et al. Parental occupation is a risk factor for childhood wheeze and asthma. Eur Respir J 2010;35:987-93.

16. Braun-Fahrlander C, Riedler J, Herz U, et al. Environmental exposure to endotoxin and its relation to asthma in school-age children. N Engl J Med 2002;347:869-77.

17. Olsen J, Melbye M, Olsen SF, et al. The Danish National Birth Cohort-its background, structure and aim. Scand J Public Health 2001;29:300-7.

18. Kennedy SM, Le MN, Choudat D, et al. Development of an asthma specific job exposure matrix and its application in the epidemiological study of genetics and environment in asthma (EGEA). Occup Environ Med 2000;57:635-41.

19. Karvonen AM, Hyvarinen A, Gehring U, et al. Exposure to microbial agents in house dust and wheezing, atopic dermatitis and atopic sensitization in early childhood: a birth cohort study in rural areas. Clin Exp Allergy 2012;42:1246-56.

20. Asher MI, Keil U, Anderson HR, et al. International study of asthma and allergies in childhood (ISAAC): rationale and methods. Eur Respir J 1995;8:483-91.

21. Li X, Sundquist K, Sundquist J. Parental occupation and risk of hospitalization for asthma in children and adolescents. J Asthma 2009;46:815-21.

22. Tagiyeva N, Anua SM, Semple S, et al. The 'take home' burden of workplace sensitizers: flour contamination in bakers' families. Environ Int 2012;46:44-9.

23. Holloway JA, Warner JO, Vance GH, et al. Detection of house-dustmite allergen in amniotic fluid and umbilical-cord blood. Lancet 2000;356:1900-2.

24. Lim RH, Arredouani MS, Fedulov A, et al. Maternal allergic contact dermatitis causes increased asthma risk in offspring. Respir Res 2007;8:56

25. Christensen BH, Thulstrup AM, Hougaard KS, et al. Occupational exposure during pregnancy and the risk of hay fever in 7-year-old children. Clin Respir J 2012.

26. Basinas I, Schlünssen V, Heederik D, et al. Sensitisation to common allergens and respiratory symptoms in endotoxin exposed workers: a pooled analysis. Occup Environ Med 2012;69:99-106.

27. Jenkins MA, Hopper JL, Flander LB, et al. The associations between childhood asthma and atopy, and parental asthma, hay fever and smoking. Paediatr Perinat Epidemiol 1993;7:67-76.

28. Scholtens $\mathrm{S}$, Wijga $\mathrm{AH}$, Brunekreef $\mathrm{B}$, et al. Maternal overweight before pregnancy and asthma in offspring followed for 8 years. Int $J$ Obes (Lond) 2010;34:606-13.

29. Laerum BN, Svanes C, Wentzel-Larsen T, et al. Young maternal age at delivery is associated with asthma in adult offspring. Respir Med 2007; 101:1431-8

30. Lodrup Carlsen KC, Carlsen KH. Effects of maternal and early tobacco exposure on the development of asthma and airway hyperreactivity. Curr Opin Allergy Clin Immunol 2001; $1: 139-43$.

31. Benn CS, Thorsen $\mathrm{P}$, Jensen JS, et al. Maternal vaginal microflora during pregnancy and the risk of asthma hospitalization and use of antiasthma medication in early childhood. J Allergy Clin Immunol 2002:110:72-7.

32. Shaheen SO, Newson RB, Henderson AJ, et al. Prenatal paracetamol exposure and risk of asthma and elevated immunoglobulin E in childhood. Clin Exp Allergy 2005; 35:18-25.

33. Lowe AJ, Carlin JB, Bennett CM, et al. Do boys do the atopic march while girls dawdle? J Allergy Clin Immunol 2008;121:1190-5.

34. Svanes C, Omenaas E, Heuch JM, et al. Birth characteristics and asthma symptoms in young adults: results from a population-based cohort study in Norway. Eur Respir J 1998;12:1366-70.

35. Nohr EA, Frydenberg M, Henriksen TB, et al. Does low participation in cohort studies induce bias? Epidemiology 2006;17:413-18.

36. Greene N, Greenland S, Olsen J, et al. Estimating bias from loss to follow-up in the Danish National Birth Cohort. Epidemiology 2011;22:815-22.

37. Hermann C, De Fine ON, Host A, et al. Prevalence, severity and determinants of asthma in Danish 5-year-olds. Acta Paediatr 2006;95:1182-90.

38. Bergmann RL, Edenharter G, Bergmann KE, et al. Atopic dermatitis in early infancy predicts allergic airway disease at 5 years. Clin Exp Allergy 1998;28:965-70.

39. Benn CS, Benfeldt E, Andersen PK, et al. Atopic dermatitis in young children: diagnostic criteria for use in epidemiological studies based on telephone interviews. Acta Derm Venereol 2003;83: 347-50. 